\title{
Norois
}

Environnement, aménagement, société

\section{Une étude de brise de mer dans la région caennaise}

Vers une aide à la prévention de la pollution photochimique en milieu littoral

A sea breeze study in the Region of Caen. Towards photochemical pollution

safety in coastal area

\section{Aurélie Dudouit}

\section{(2) OpenEdition}

Journals

Édition électronique

URL : https://journals.openedition.org/norois/721

DOI : 10.4000/norois.721

ISBN : 978-2-7535-1541-3

ISSN : $1760-8546$

Éditeur

Presses universitaires de Rennes

\section{Édition imprimée}

Date de publication : 1 décembre 2004

Pagination : $31-45$

ISBN : 978-2-7535-0081-5

ISSN : 0029-182X

\section{Référence électronique}

Aurélie Dudouit, « Une étude de brise de mer dans la région caennaise », Norois [En ligne], 193 | 2004/4 mis en ligne le 14 août 2008, consulté le 13 janvier 2022. URL : http://journals.openedition.org/norois/ 721 ; DOI : https://doi.org/10.4000/norois.721

Ce document a été généré automatiquement le 13 janvier 2022.

(c) Tous droits réservés 


\section{Une étude de brise de mer dans la région caennaise}

Vers une aide à la prévention de la pollution photochimique en milieu littoral

A sea breeze study in the Region of Caen. Towards photochemical pollution

safety in coastal area

Aurélie Dudouit

\section{NOTE DE L'ÉDITEUR}

Cet article a été reçu le 3 février 2004 et définitivement accepté le 2 septembre 2004.

Remerciements : Je tiens à remercier Armel Coudé, Stéphane Costa et Olivier Planchon pour leur aide efficace et leurs précieuses suggestions.

\section{Introduction}

1 Ce travail s'inscrit dans le cadre d'une thèse menée actuellement sur les risques de pollution photochimique dans la région caennaise (Calvados, France) en période de brise de mer et de terre (fig. 1). La prévision et la prévention des risques de pollution atmosphérique sont étroitement dépendantes des conditions aérologiques d'échelles synoptiques, régionales et locales. En effet, dans le cas d'une émission polluante de l'air, le transport et la dispersion de la substance sont soumis aux circulations atmosphériques dont l'échelle varie entre plusieurs milliers de kilomètres et quelques mètres. Les mouvements anticycloniques ou dépressionnaires peuvent déplacer cette matière sur une échelle planétaire tandis que la turbulence de micro-échelle la disperse dès son émission. Entre ces deux échelles extrêmes, il existe des circulations régionales dont l'extension spatiale est comprise entre quelques kilomètres et quelques centaines de kilomètres. Les recherches sur la relation entre la météorologie et la pollution 
atmosphérique sont nombreuses mais à ce jour, en Basse-Normandie, aucune n'a traité de façon exhaustive le rôle des brises de mer sur la qualité de l'air. Or, comme toute région littorale, la Basse-Normandie est soumise à ces circulations qui déterminent fortement l'occurrence et l'intensité des épisodes de pollution atmosphérique en général, et les problèmes de pollution photochimique (ozone) en particulier.

2 La brise peut être définie comme un vent alternatif (brise diurne et brise nocturne), d'origine thermique et d'échelle locale dont l'extension horizontale peut varier de quelques centaines de mètres à plusieurs dizaines de kilomètres (Pedelaborde, 1985 ; Simpson, 1994). Si la brise de mer est aisément identifiable dans la région caennaise, d'une manière générale, la brise de terre est un phénomène rare dans notre secteur d'étude (des précisions seront apportées dans la suite de l'article).

3 La première partie de la recherche en cours a consisté à établir une base de données sur les journées de brise de mer dans le but de mieux connaitre et comprendre leur fonctionnement sur le littoral de la Côte de Nacre. Sa finalité sera de prévoir ces circulations d'échelle régionale pour prévenir les pics d'ozone associés.

Alors que les circulations de brise sont souvent étudiées de manière théorique à partir de la modélisation (Neumann et Mahrer, 1971), ce travail propose une approche originale et géographique pour caractériser, étudier et prévoir les journées de brise. En effet, le but de l'étude n'est pas de simuler des journées théoriques de brises de mer sur la Côte de Nacre mais de comprendre, à partir de cas concrets et de mesures réalisées sur plusieurs sites, leurs fonctionnements.

5 Il s'agit principalement ici de présenter une méthode de détection des journées de brise de mer à partir de données horaires de plusieurs paramètres météorologiques mesurés au sol dans une région littorale à la topographie différenciée. Cette méthode, reposant sur un logiciel, est fondée sur l'utilisation de filtres automatiques construits à partir de plusieurs caractéristiques significatives des brises de mer.

6 Cet article traite, en premier lieu, de l'étude des données météorologiques anciennes, entre 1993 et 2002 de la station de Caen-Carpiquet appartenant au réseau de MétéoFrance. Dans un deuxième temps, les données enregistrées par nos soins au cours d'une campagne de mesures réalisée pendant la période estivale de 2003 et celles du réseau de Météo-France sont étudiées. Enfin, une synthèse de l'étude des journées de brise de mer est proposée.

\section{Méthode}

7 Le littoral de la Côte de Nacre (fig. 1) fournit un espace pertinent d'application car les brises de mer y apparaissent de manière récurrente et les réseaux de mesures météorologiques y sont de qualité.

8 Une analyse, sur la période antérieure 1993-2002, des séries de données météorologiques horaires de la station du Centre Départemental Météorologique de Caen-Carpiquet a été réalisée dans le but de constituer un échantillon de journées soumises à la brise de mer.

9 La station de Caen-Carpiquet, située à une quinzaine de kilomètres du littoral peut paraître un peu éloignée pour étudier l'établissement des brises de mer. La station de Bernières située sur le littoral aurait été, en effet, préférable pour détecter les journées de brise. Mais elle a été installée trop tardivement (août 2001) pour être utilisée. 
Cependant, il existe des relations significatives entre les classes de vent (test du significatif à moins de $0,1 \%$ après partition en seize directions et une de vent nul) et les vitesses de vent (coefficients de corrélation significatifs à moins de $0,1 \%$ ) à CaenCarpiquet et à Bernières en période de brise de mer. La station de Caen-Carpiquet a donc une bonne représentativité de la direction et de la vitesse du vent par rapport au littoral et peut être utilisée pour l'étude. De plus, la station de Caen-Carpiquet a été choisie parce qu'elle dispose de la base de données horaires la plus importante du Calvados.

Figure 1 : Présentation de la zone d'étude : la région caennaise Presentation of the study area : the region of Caen

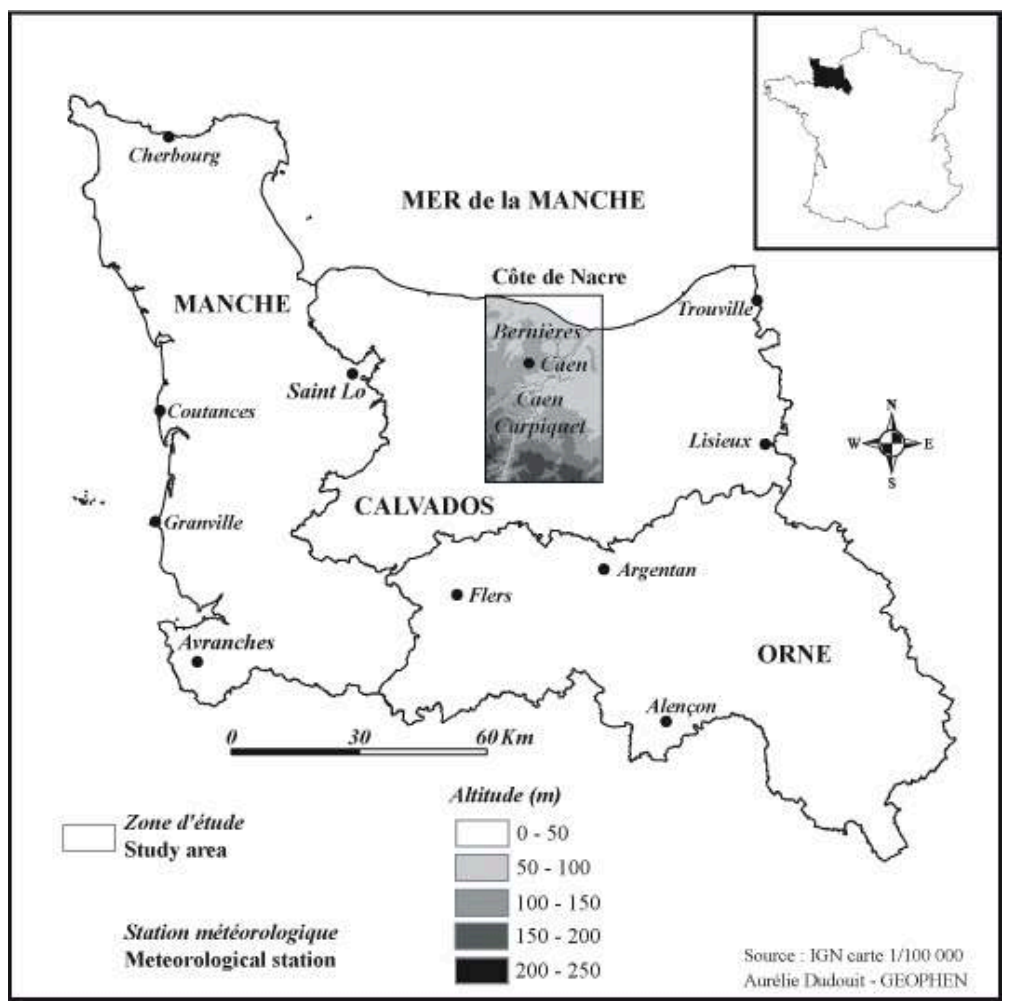

L'étude a porté uniquement sur une période de dix ans parce qu'il n'existe pas d'enregistrement horaire de la direction et de la vitesse du vent à la station de CaenCarpiquet avant 1993. De ce fait, les journées de brise de mer n'auraient pu être convenablement étudiées avant cette date. En effet, pour ce type de phénomènes locaux, l'utilisation de données enregistrées à un pas de temps fin (horaire au minimum) est indispensable pour déterminer précisément les horaires (renverse du vent, intensité maximale, etc.) et les modifications induites par le passage du front de brise (Leriche et al., 1998).

11 Le dépouillement des journées de brise de mer a été réalisé entre le mois d'avril et le mois de septembre, période la plus favorable à ces circulations. En effet, en Europe du Nord, la saison chaude est relativement courte et la période favorable au développement des circulations de brise est centrée sur le printemps et l'été. Alors que la température de l'air s'élève, la Manche et la Mer du Nord restent fraîches, ce qui stabilise les basses couches de l'atmosphère et favorise une anticyclogenèse des îles Britanniques aux pays Baltes (Kendrew, 1961 ; Planchon, 1997). 
La détection des journées de brise étant particulièrement longue, nous avons développé un logiciel «Breeze » à partir des premières observations et en prenant en compte de nombreuses études menées sur les caractéristiques des circulations de brise (Neumann et Mahrer, 1971 ; Lyons et Olsson, 1972 ; Brittain, 1978 ; Escourrou, 1978 ; Pedelaborde, 1985a 1985b ; Simpson, 1994 ; Borne et al., 1998 ; Leriche et al., 1998 ; Dudouit, 2001).

Ce logiciel «Breeze » effectue plusieurs opérations successives de sélection à partir des données horaires de la pression atmosphérique, de la direction et de la vitesse du vent et à partir des données journalières des précipitations. Le logiciel a été défini en cinq filtres devant être tous vérifiés pour que la journée soit validée comme étant une journée possible de brise de mer (fig. 2). Ces filtres reflètent les principales caractéristiques météorologiques d'une brise de mer, à savoir une situation anticyclonique ou de marais barométrique, un vent synoptique faible pour que la brise de mer puisse s'installer, un changement de la direction du vent accompagné d'une augmentation de la vitesse du vent ou une seule augmentation de la vitesse du vent (cas où le vent synoptique est de même direction que celle de la brise de mer le matin).

Figure 2 : Description des filtres de la méthode de détection des journées de brise sur lesquels le logiciel « Breeze » a été développé

Description of the filters used in the software "Breeze" of the sea breezes detection

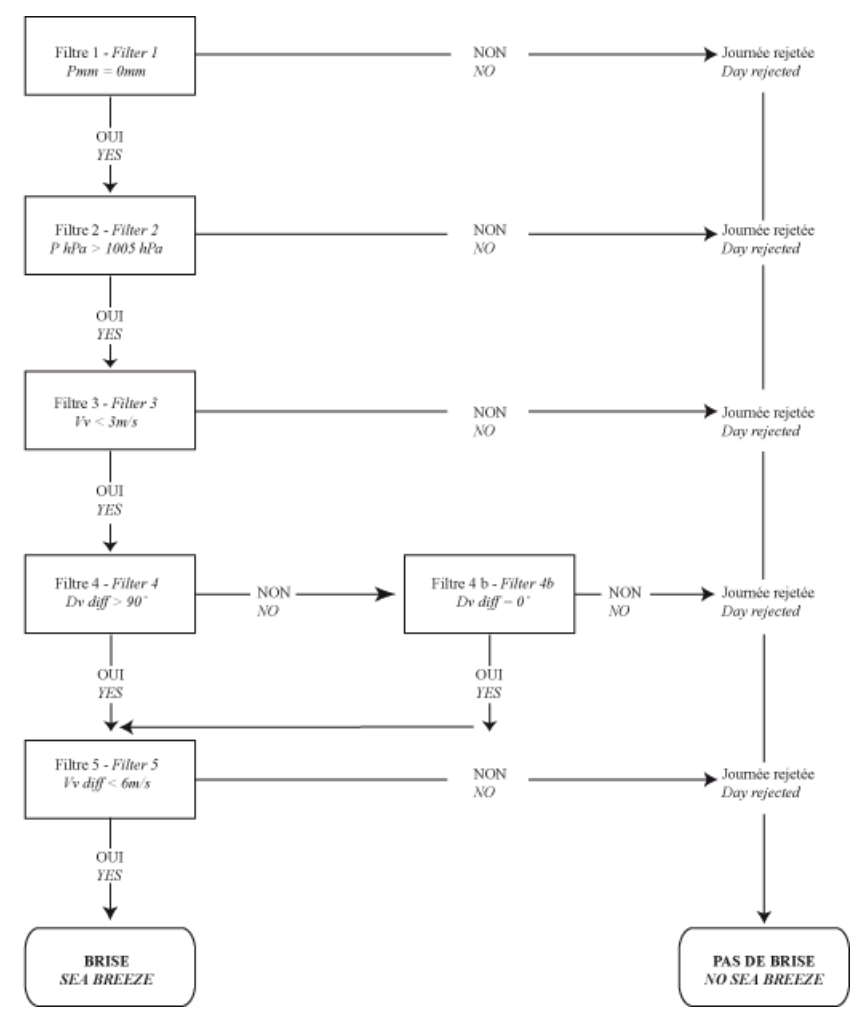

Avec Pmm : Précipitations en millimètres, $\mathrm{P}$ hPa : Pression atmosphérique en Hectopascal, Vv : Vitesse du vent en $\mathrm{m} / \mathrm{s}, \mathrm{Vv}$ diff : Changement de la vitesse du vent au cours de la journée considérée, Dv : Direction du vent en degrés, Dv diff : Changement de la direction du vent au cours de la journée considérée.

With Pmm : Rainfall in millimeters, $P h P a$ : atmospheric pressure in $h P a, V_{v}$ : wind speed in $\mathrm{m} / \mathrm{s}, \mathrm{Vv}$ diff: Change in wind speed during the day, Dv : wind direction in degrees, Dv diff: Change in wind direction during the day.

- Le premier filtre consiste à supprimer toutes les journées pluvieuses, c'est-à-dire les journées où le total des précipitations journalières est supérieur à zéro millimètre. 
- Le deuxième filtre, rejette toutes les journées à tendance dépressionnaire pour lesquelles la pression atmosphérique est inférieure à 1005 Hectopascals à un moment de la journée.

- Le troisième filtre consiste à éliminer les journées pour lesquelles la vitesse du vent est trop importante le matin pour qu'une brise de mer puisse se lever, c'est-à-dire lorsque la vitesse du vent est supérieure à $3 \mathrm{~m} / \mathrm{s}$.

- Les quatrième et cinquième filtres, activés simultanément, permettent de vérifier si la brise de mer s'est levée, c'est-à-dire si un changement de la direction du vent d'au moins $90^{\circ}$ de la terre vers la mer au cours de la journée associé à une augmentation de la vitesse du vent de moins de $6 \mathrm{~m} / \mathrm{s}$ s'est produit (fig. 3). À ce stade de la sélection, deux autres filtres peuvent être activés, les quatrième et cinquième filtres bis ; ils sélectionnent les journées de brise de mer pour lesquelles il n'y a pas eu de changement de direction du vent mais une augmentation de la vitesse du vent toujours inférieure à $6 \mathrm{~m} / \mathrm{s}$. Il s'agit des cas où le vent synoptique le matin est de la même direction que la brise de mer. L'existence ou non d'une renverse en fin de journée n'est pas un critère de sélection de brise puisque sur nos côtes la direction du vent peut se stabiliser au nord-est ou au nord-ouest.

Figure 3 : Caractéristiques de la direction et de la vitesse du vent d'une journée de brise sélectionnée par le logiciel « Breeze ». Données enregistrées à la station de Caen-Carpiquet le 21 juillet 1996

Characteristics of wind speed and wind direction for a selected day as a sea breeze by the software "Breeze". Data were collected on the 21 July 1996 at Caen-Carpiquet
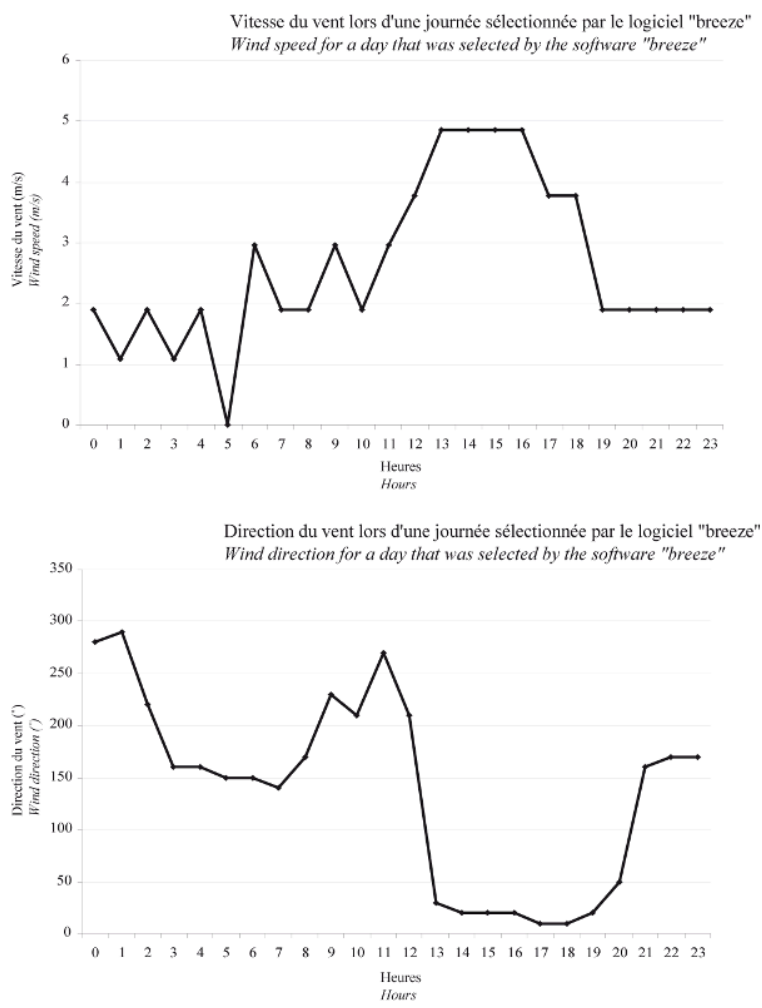

Les journées de brise retenues sont ensuite validées avec les enregistrements horaires de la température de l'air $\left({ }^{\circ} \mathrm{C}\right)$ et de l'humidité relative (\%). Le critère retenu est une nette diminution de la température de l'air au moment de l'apparition de la brise de mer accompagnée d'une augmentation de l'humidité relative. Cent soixante et une journées de brise ont ainsi été détectées.

Le premier intérêt d'un tel logiciel est la sélection rapide des journées de brise à partir d'une base de données importante (7 320 données à analyser). Le second est de limiter, 
dans un premier temps, le coût de la base de données nécessaire à l'étude d'un phénomène d'échelle temporelle fine. Cette méthode permet à partir de trois paramètres enregistrés au pas de temps horaire, de dégager les journées de brise pour lesquelles d'autres données peuvent être ensuite demandées pour analyser précisément le phénomène. Cependant, quelques critiques peuvent être faites quant au nombre restreint de paramètres utilisés dans la méthode de détection des journées de brise par filtres automatiques. Il est en effet dommage que pour la région caennaise des données sur les conditions aérologiques en altitude (les radiosondages les plus proches sont réalisés à Brest ou à Trappe) et sur la température de la mer (l'étude de la différence de température entre la mer et l'air étant importante dans la mise en place des circulations de brise) n'existent pas. Ces données auraient pu être utilisées dans des filtres supplémentaires et ainsi améliorer la méthode.

L'étude des données météorologiques entre 1993 et 2002 de la station de CaenCarpiquet a permis de déterminer la fréquence des brises de mer et leurs caractéristiques météorologiques et temporelles. Mais leur fonctionnement spatial dans la région caennaise n'a pas encore été défini. Ainsi, une campagne de mesures a été mise en place dans le but d'explorer des zones non documentées aujourd'hui et de déterminer l'extension horizontale de la brise dans la région caennaise en tenant compte de la topographie. En étudiant les données de Météo-France (Caen-Carpiquet) et celles acquises sur le terrain par nos soins, les caractéristiques spatio-temporelles des circulations de brise ont ainsi pu être analysées.

Pour mener à bien cette campagne de terrain, du matériel de mesures météorologiques a été installé pendant une période de six mois ( $1^{\mathrm{er}}$ avril-30 septembre 2003), le long de quatre transects, sur différents sites définis de manière à refléter les effets éventuels de la topographie sur les écoulements (fig. 4).

Pour réaliser les mesures météorologiques, deux stations automatiques «LeaderYoung", six stations automatiques "Davis » et une station automatique "Pulsonic » ont été utilisées. Des mesures de la direction et de la vitesse du vent, de la température de l'air, de l'humidité relative, de la pression atmosphérique et du rayonnement global ont ainsi été réalisées au pas de temps quart horaire. 
Figure 4 : Réseau de mesures météorologiques mis en place dans la région caennaise durant la période estivale 2003

Location of the meteorological stations used for the study during the 2003 summer in the region of Caen

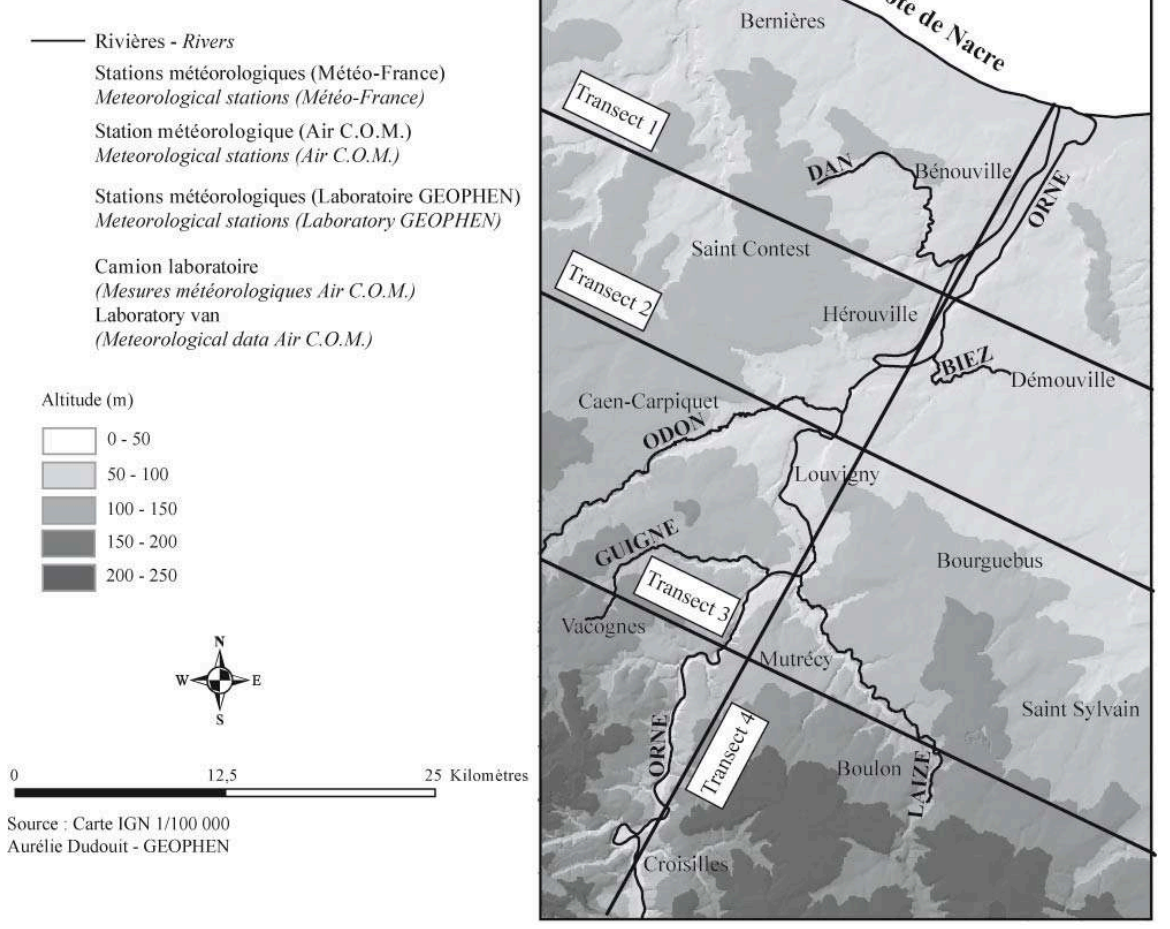

\section{Résultats}

\section{Étude des circulations de brise de mer sur la Côte de Nacre depuis 1993}

L'étude des journées de brise entre 1993 et 2002 a montré que ces circulations d'échelle régionale soufflaient plus ou moins fréquemment d'une année sur l'autre mais apparaissaient tous les ans. Parmi ces journées, $28 \%$ ont été enregistrées au mois de juillet et $20 \%$ au mois d'août (fig. 5).

Le caractère saisonnier de la brise de mer montre que l'apparition de cette circulation dépend de certaines situations météorologiques plus fréquentes en périodes printanière et estivale. Un inventaire des différentes situations synoptiques rencontrées lors des 161 journées de brise retenues a donc été dressé. Ce travail est basé sur l'analyse des bulletins météorologiques européens en surface et en altitude à $850 \mathrm{hPa}$. Pour chacun de ces deux niveaux d'analyse trois grandes classes de situation ont été distinguées: les situations anticycloniques et de dorsales anticycloniques, les situations de marais barométrique et les situations dépressionnaires à faible gradient. Les situations anticycloniques ont été le plus souvent observées. C'est pourquoi ce travail a été affiné en divisant cette classe en trois groupes selon la position longitudinale et latitudinale de l'anticyclone. Nous avons ainsi distingué les journées où l'anticyclone était situé sur l'Europe du Nord, celles où l'anticyclone était positionné sur l'Europe moyenne et celles où l'anticyclone se trouvait au-dessus de l'Atlantique. Les résultats obtenus sont conformes à ceux de F. Damato et al. (2003). Les journées de 
brise apparaissent majoritairement lorsque l'anticyclone est localisé sur l'Europe du Nord.

Figure 5 : Nombre et fréquence mensuelle des journées de brise de mer retenues pendant la période estivale (avril-septembre) durant la période 1993-2002 à la station de Caen-Carpiquet Number and monthly frequency of sea breeze days in summer (April-September) during the period 1993-2002 at Caen-Carpiquet
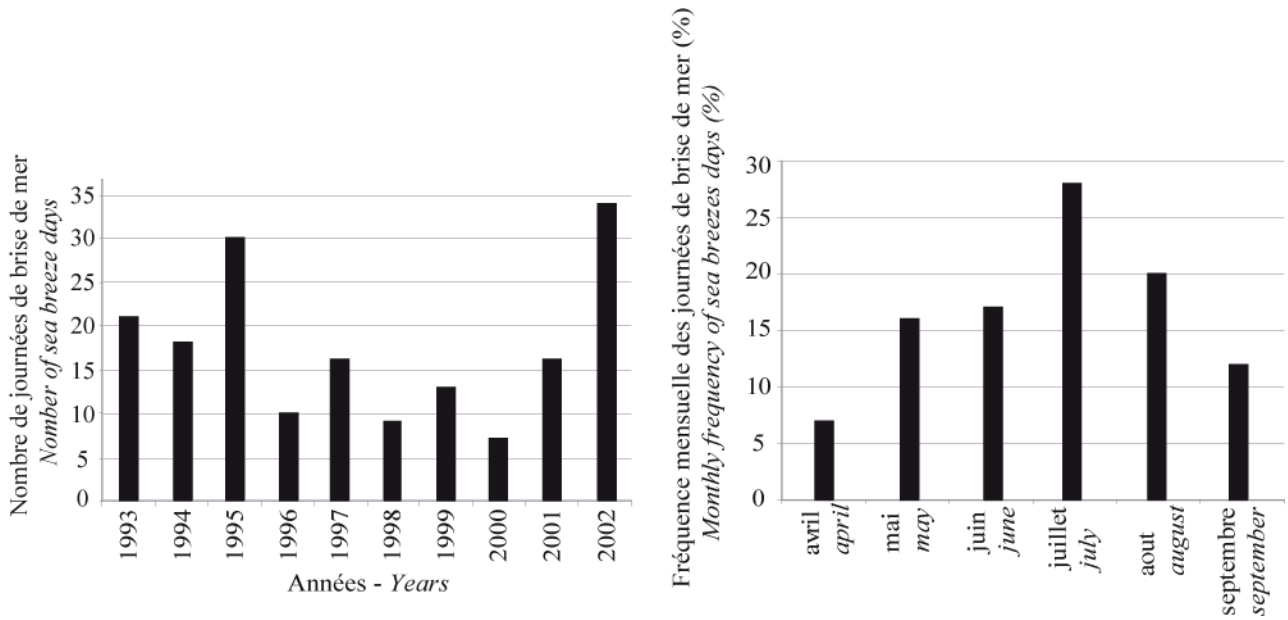
continent, selon une direction proche de la perpendiculaire au littoral en début d'après-midi, oblique d'environ $15^{\circ}$ de part et d'autre de la perpendiculaire au rivage le matin et le soir (Janoueix-Yacono, 1995). La Côte de Nacre étant orientée $\left(100^{\circ}-280^{\circ}\right)$, la brise de mer devrait souffler d'une direction de $10^{\circ}$ ou de $350^{\circ}$. Or, en fait, une brise de mer ou de terre souffle plutôt d'une direction comprise dans un intervalle de plusieurs dizaines de degrés de part et d'autre de la perpendiculaire de la côte. Ce phénomène s'explique parce qu'une brise "idéale » " pure » n'existe pas (Carrega, 1995). En effet, une circulation de brise est influencée par un vent synoptique même faible (inférieur à $3 \mathrm{~m} / \mathrm{s}$ ). Si les deux flux sont thermiquement proches et les directions assez semblables, ils tendent alors à se combiner. La coexistence de deux processus n'ayant pas exactement la même direction initiale explique alors que la direction de la brise puisse être comprise dans un intervalle de plusieurs dizaines de degrés de part et d'autre de la perpendiculaire de la côte.

Pour faciliter le discours, nous avons convenu qu'une brise de mer de nord-est est une brise qui souffle d'une direction comprise entre $05^{\circ}$ et $45^{\circ}$ et qu'une brise de mer de nord-ouest est une brise qui souffle d'une direction comprise entre $315^{\circ}$ et $355^{\circ}$.

L'évolution de la direction du vent au cours des renverses du matin et du soir (apparition et disparition de la brise de mer) dépend de l'orientation du vent synoptique (fig. 6). Ainsi, si le vent synoptique souffle d'une direction comprise entre $130^{\circ}$ et $175^{\circ}$ (sud-est), la brise s'établit au nord-est. De même, par vent synoptique de direction comprise entre $185^{\circ}$ et $230^{\circ}$ (sud-ouest), la brise de mer est de nord-ouest. On assiste ainsi dans un premier temps à une confrontation entre le vent synoptique et la brise naissante (Leriche et al., 1998). Plus tard, lorsque la circulation de brise est bien établie, l'influence du vent synoptique ne se fait quasiment plus sentir. En fin de brise de mer, la direction du vent évolue de nouveau et plusieurs cas de figures peuvent alors se présenter (fig. 6). Si le vent synoptique est nul voire de très faible vitesse, l'orientation du vent peut soit rester au nord-est ou au nord-ouest, soit continuer à 
évoluer jusqu'au secteur est-sud-est dans le cas d'une brise de mer de nord-est ou jusqu'au secteur ouest-sud-ouest dans le cas d'une brise de nord-ouest. Quelques cas ont également montré qu'une légère brise de terre de sud-sud-est ou de sud-sud-ouest pouvait se lever en fin de soirée. Mais, de manière générale, la brise de terre est un phénomène très rare dans notre secteur d'étude. Si le vent synoptique n'est pas nul, la direction observée lorsque la brise cesse de souffler en fin d'après-midi ou en début de soirée est celle du vent synoptique observé le matin avant le déclenchement de la brise de mer.
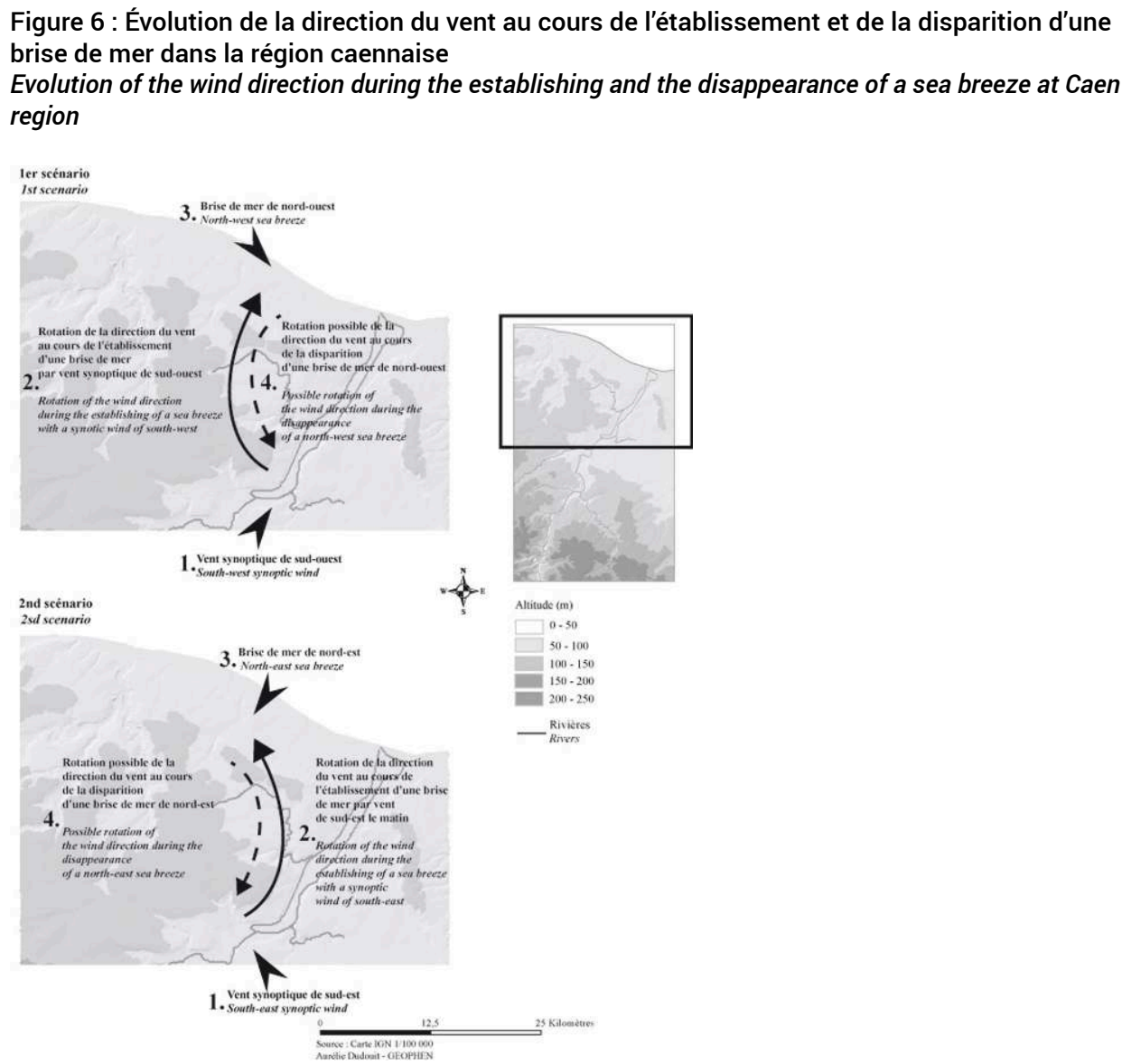

Outre l'évolution de la direction du vent durant les périodes de renverse, les séquences de journée de brise ont été étudiées, c'est-à-dire le nombre de jours consécutifs pendant lesquels la brise a soufflé, caractère des circulations de brise primordial à prendre en compte dans l'étude de la qualité de l'air. Sur la période d'étude analysée (1993-2002) à Caen-Carpiquet, six séquences de jours consécutifs de brise ont été dégagées : des séquences de deux, trois, quatre, cinq, six et sept jours. $52 \%$ des 161 journées de brise retenues sont comprises dans ces séquences de brise. De même que l'apparition des brises de mer est plus fréquente aux mois de juillet et août, elles s'organisent davantage en séquences durant ces mêmes mois.

La vitesse maximale atteinte lors d'une journée de brise de mer est généralement comprise entre 5 et $7 \mathrm{~m} / \mathrm{s}$ et est toujours inférieure à $10 \mathrm{~m} / \mathrm{s}$. Il existe une variabilité inter mensuelle de la vitesse maximale de la brise de mer, les valeurs les plus élevées étant enregistrées durant les mois de juillet et août, période la plus chaude de l'année. 
De plus, le vent synoptique influence également la vitesse des brises de mer. En effet, plus il est important, plus la brise de mer soufflera fort quelle que soit sa direction. Selon plusieurs études, il semblerait que les vents synoptiques de secteur nord pourraient ajouter une composante au vecteur vitesse du courant de brise, alors que les vents synoptiques de secteur sud renforceraient le vortex et donc l'intensité de la brise par effet de friction sur le front de brise (Estoque, 1962; Arritt, 1993). Il semblerait également qu'il existe une relation entre les types de brise et la vitesse de vent. Sur la Côte de Nacre, les brises de nord-ouest sont, en effet, plus faibles que celles de nord-est.

La brise de mer ayant un rythme nycthéméral, son apparition et sa disparition sont liées aux horaires de lever et de coucher du soleil en fonction de la saison. Concernant l'étude des heures moyennes mensuelles d'apparition et de disparition de la brise de mer avec les heures moyennes mensuelles du lever et du coucher du soleil, la brise de mer apparaît en moyenne 6 heures après le lever du soleil et disparaît en moyenne 3 heures avant le coucher du soleil quel que soit le mois considéré. Mais, la durée du jour n'est pas le seul facteur qui détermine les horaires des brises de mer. En effet, la direction du vent synoptique influence fortement l'heure d'apparition de la brise de mer parce qu'il déplace la zone de gradient de températures par rapport au rivage (Leriche et al., 1998). Quant à la durée de la brise de mer, elle dépend bien entendu des horaires du lever et du coucher du soleil mais également de l'évolution de la vitesse et de la direction du vent synoptique pendant le développement de la brise de mer.

\section{Dynamique spatio-temporelle des circulations de brise de mer dans la région caennaise durant la période estivale 2003}

L'été 2003 a fait l'objet d'une campagne de terrain afin de caractériser l'évolution spatiale et temporelle des circulations de brise de mer dans la région caennaise. Cette étude fait suite à l'analyse des données météorologiques durant la période 1993-2002 qui a mis en évidence la fréquence et l'occurrence des brises de mer.

Avant toute chose, il convient de préciser que la période de canicule de l'été 2003, exceptionnelle par sa durée et son intensité, n'a pas été sans conséquences dans de multiples domaines : la ventilation et la pollution photochimique.

Concernant les circulations de brise de mer, la situation météorologique (fig. 7), identique pendant toute la canicule (entre le 4 et le 12 août 2003), aurait dû entraîner des journées de brise puisqu'elles apparaissent majoritairement lorsque l'anticyclone est situé sur l'Europe du Nord. 
Figure 7 : Situation synoptique durant la canicule de l'été 2003 : exemple du 5 août 2003 Synoptic situation during the heatwave of the summer 2003 : exemple of the 8 mai 2003

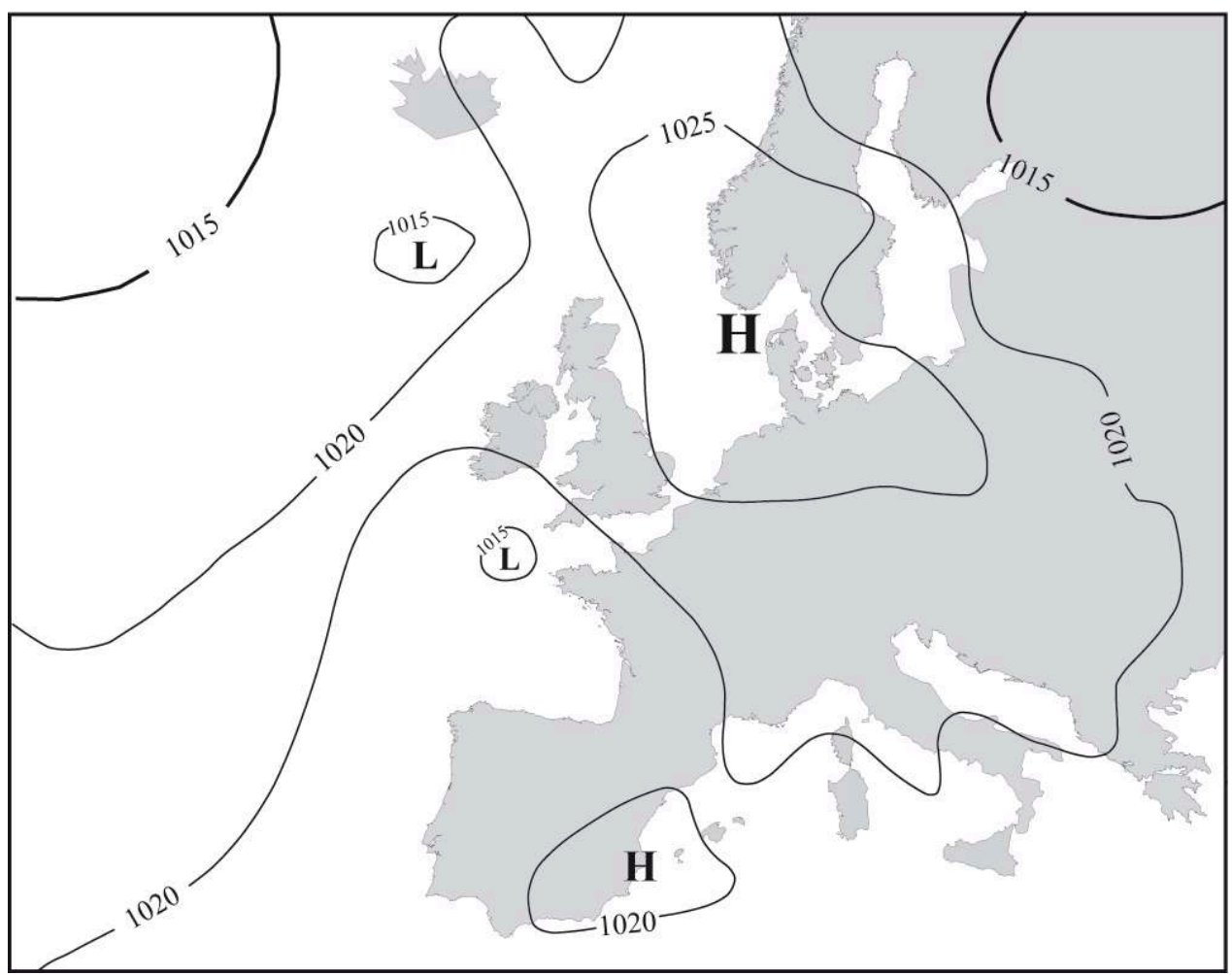

Or, la fréquence et l'intensité des vents de direction nord-ouest et nord-est (directions majoritaires de la brise de mer dans la région caennaise (fig. 6) ont été très faibles durant la canicule (fig. 8). Il n'y a donc pas eu de journées de brise de mer comme on a pu en observer durant la période 1993-2002 (vent de direction nord-ouest ou nord-est de vitesse soutenue : entre 5 et $7 \mathrm{~m} / \mathrm{s}$ ).

Notre campagne de terrain est donc à replacer dans un contexte climatique très particulier et inhabituel. Les enregistrements de cette période seront donc à nuancer par rapport aux résultats obtenus à la suite de l'étude des données météorologiques antérieures (1993-2002).

Durant la campagne de mesures effectuée entre le $1^{\mathrm{er}}$ avril et le 30 septembre 2003, 44 journées de brise de mer ont été enregistrées. $68 \%$ de ces journées étaient des brises de nord-est. Il semblerait, comme on pouvait s'y attendre, qu'elles ne pénètrent pas dans les terres de manière homogène. $40 \%$ des journées de brise ne se sont pas faits ressentir aux stations du sud de la zone d'étude (Vacognes, Mutrécy, Boulon, SaintSylvain et (roisilles), stations situées à une distance d'au moins dix-huit kilomètres du littoral. Et, dans $11 \%$ des cas, la renverse du soir, c'est-à-dire l'évolution de la direction du vent au moment de la disparition de la brise de mer (retour à la direction du vent synoptique du matin), observée au sud de la région caennaise n'a pas été enregistrée aux stations situées proche de la côte (le vent s'est stabilisé dans la direction de la brise de mer). En moyenne, sur la totalité de la période des mesures, la brise de mer a eu une durée de sept heures au nord de la zone expérimentale et de trois heures au sud. De plus, 35 des 44 journées de brise enregistrées durant la période estivale 2003 étaient enregistrées en séquence de deux, trois ou quatre jours, ce qui représente une proportion de journées de brises organisées en séquence beaucoup plus importante que 
ce qui avait été montré à la suite de l'étude des données météorologiques entre 1993 et 2002.

Figure 8 : Roses des vents de la station de Bénouville durant la totalité de la campagne de mesures (A.) et lors de la canicule (B.)

Compass cards of the station of Benouville during the campain of measurements (A.) and during the heatwave (B.)

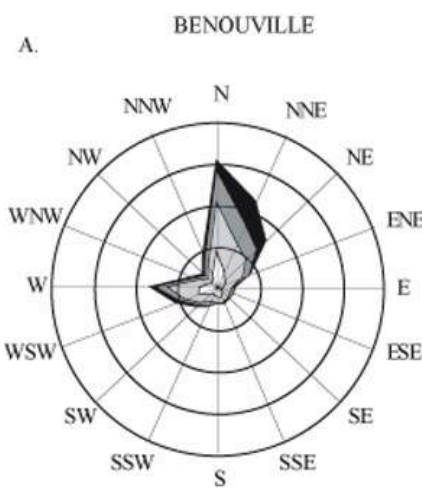

Vent nul - No wind : $27 \%$

Vitesse movenne du vent ả $10 \mathrm{~m}: 2,5 \mathrm{~m} / \mathrm{s}$ Mean wind speed at 10 meters : $2,5 \mathrm{~m} / \mathrm{s}$

Nombre de cas observés : 8965 Nomber of observed cases : 8965

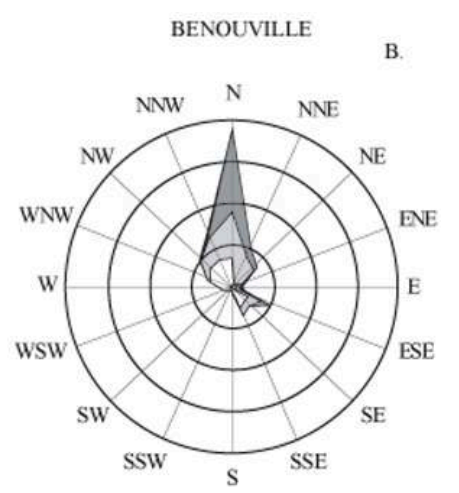

Vent nul - No wind : $25 \%$

Vitesse moyenne du vent a $10 \mathrm{~m}: 2,1 \mathrm{~m} / \mathrm{s}$ Mean wind speed at 10 meters : $2,1 \mathrm{~m} / \mathrm{s}$

Nombre de cas observés : 768 Nomber of observed cases : 768 
Figure 9 : Évolution moyenne du front de brise durant la période estivale de 2003 au sein de la région caennaise

Mean evolution of the sea breeze front during the summer 2003 at the Caen region

Zone d'étude - Study area

Rivières - Rivers

Stations météorologiques (Météo-France)

Meteorological stations (Météo-France)

Station météorologique (Air C.O.M.)

Meteorological stations (Air C.O.M.)

Stations météorologiques (Laboratoire GEOPHEN)

Meteorological stations (Laboratory GEOPHEN)

Camion laboratoire

(Mesures météorologiques Air C.O.M.)

Laboratory van

(Meteorological data Air C.O.M.)

Altitude (m)

$0-50$

$50-100$

$100-150$

$150-200$

$200-250$

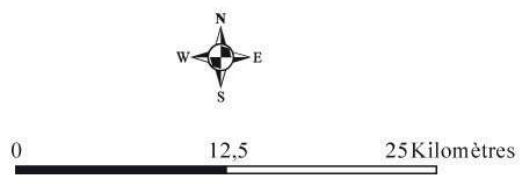

Source : Carte IGN 1/100 000 Aurélie Dudouit - GEOPHEN

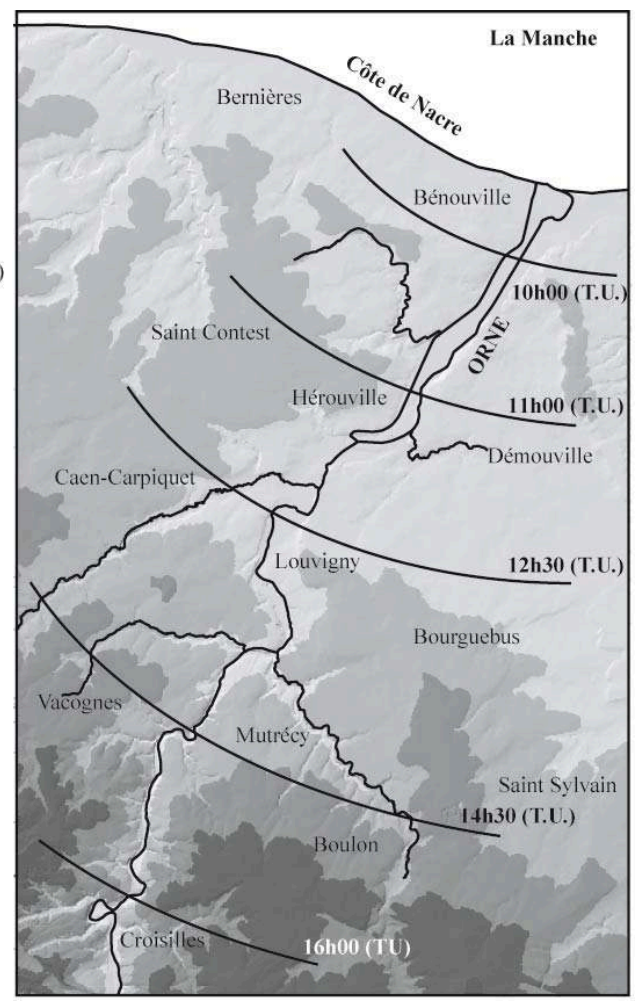

\section{Conclusions et perspectives}

Cette étude a permis, à partir d'une méthode simple de sélection, de constituer un échantillon de journées de brise. En effet, grâce au logiciel «Breeze», les caractéristiques principales de ces journées (période d'occurrence, fréquences d'apparition, horaires et intensité du phénomène, évolution de la direction du vent au cours d'une journée de brise, organisation cyclique des journées de brise) ont ainsi pu être définies en un site: Caen-Carpiquet. La campagne de mesures a, quant à elle, permis de caractériser les journées de brise en différents sites et notamment de comprendre l'évolution du front de brise sur un espace à topographie différenciée (Plaine de Caen/hauteurs bocaines).

Cependant, le rôle exact de la topographie sur les circulations de brises n'a pas encore été suffisamment mis en évidence dans la région caennaise. De plus, pour l'exercice de la prévision, les situations synoptiques devront être plus précisément étudiées pour connaître leur influence ou non sur le type de brise. Il a été montré par ailleurs que l'évolution de la direction du vent au cours de l'établissement et de la disparition de la brise de mer avait une importance pour la compréhension de la qualité de l'air.

Outre l'aspect de recherche fondamentale, il existe un intérêt appliqué à cette recherche puisque ce travail portant sur les relations brise de mer/ozone peut servir d'outil d'aide à la décision dans la prévention des risques sanitaires et environnementaux liés aux fortes concentrations d'ozone. Le but définitif de l'étude sera de produire une carte d'aléa aux risques de pollution par l'ozone par circulation de 
brise de mer. Cet outil pourra servir à définir des stratégies de gestion locale de la qualité de l'air relative au problème de l'ozone.

\section{BIBLIOGRAPHIE}

ARRITT (R. W.), 1993. - «Effects of the large-scale flow on characteristic features of the sea breeze », Journal of applied Meteorology, n 32, p. 116-125.

BORNE (K.), CHEN (D.), NUnEz (M.), 1998. - « A method for finding sea breeze days under stable synoptic conditions and its application to the Swedish west coast ", International Journal of Climatology, $\mathrm{n}^{\circ} 18$ (8), p. 901-914.

BRITTAIN (O. W.), 1978. - Forecasting sea-breezes at Eskmeals', Meteorol. Mag., n 107, p. 88-96.

CARREGA (P.), 1995. - Une procédure d'identification automatique et en temps réel du régime de vent, Publication de l'AIC, $n^{\circ}$ 8, p. 13-21.

DAmato (F.), PlanCHon (O.), Dubreuil (V.), 2003. - « A remote sensing study of the inland penetration of sea breeze fronts the English Channel », Weather, $n^{\circ} 58$ (6), p. 219-226.

DuDouit (A.), 2001. - Essai de prévision locale des conditions aérologiques de surface pour la pratique de la voile, Mémoire de maîtrise, Université de Caen, $196 \mathrm{p}$.

EscourRou (G.), 1978. - Climat et types de temps en Normandie, thèse d'État, Université de Lille 3, 649 p.

EsToque (M. A.), 1962. - « The sea breeze as a function of the prevailing synoptic situation », Journal of the Atmospheric Sciences, $\mathrm{n}^{\circ}$ 19, p. 244-250.

JANOUEIX-YACONO (D.), 1995. - Rapports entre brise de mer ou de lac, structure de la couche limite planétaire et pollution atmosphérique sur les plaines littorales urbanisées, Dijon, GDR Climat et Santé CNRS, Climat, Pollution atmosphérique, Santé, Hommage à Gisèle Escourrou, p. 177-201.

KENDREW (W. G.), 1961. - The climates of the continents, London, Oxford at the Clarendon Press, Oxford University Press, $608 \mathrm{p}$.

LeRiche (P.), KeRgomard (C.), CHAPElet (P.), 1998. - Étude des brises de mer sur le littoral français de la mer du nord, Publication de l'AIC, $\mathrm{n}^{\circ}$ 11, p. 107-114.

LyONS (W. A.), OLSSON (L. E.), 1972. - « The climatology and prediction of the Chicago lake breeze », Journal of Applied Meteorology, $\mathrm{n}^{\circ}$ 11, p. 1254-1272.

NEUMANn (J.), MAHRER (Y.), 1971. - « A theooretical study of the land and sea breeze circulation », Journal of the Atmospheric Sciences, $\mathrm{n}^{\circ}$ 28, p. 532-542.

PedelaboRdE (P.), 1985. - « Les brises de mer et les brises de terre », Géographie et recherche, n 54, p. 79-111.

-, 1985a. - « Les brises de mer et les brises de terre », Géographie et recherche, n 55, p. 59-108.

-, 1985b. - « Les brises de mer et les brises de terre », Géographie et recherche, n 56, p. 57-112. 
PlANCHON (O.), 1997. - Les climats maritimes dans le monde, Villeneuve-d'Ascq, Presses

Universitaires du Septentrion, $233 \mathrm{p}$.

SIMPSON (J. E.), 1994. - Sea breeze and local winds, Cambridge, Cambridge University Press, 234 p.

\section{RÉSUMÉS}

L'article présente une partie des recherches menée dans le cadre d'une thèse dont le but est de connaître et comprendre les circulations de brise de mer pour prévoir et prévenir la pollution par l'ozone dans une zone côtière située proche d'une agglomération.

La méthode de détection des journées de brise exposée ici a été développée en vue de dégager les principaux aspects de la climatologie des brises de mer sur le littoral calvadosien de la Côte de Nacre. L'étude des données météorologiques de la période 1993-2002 enregistrées à la station de Caen-Carpiquet (Météo-France) et celles obtenues au cours d'une campagne de terrain organisée par nos soins durant la période estivale de 2003 a permis de définir les fréquences d'occurrence de la brise de mer, d'une part, et l'évolution du front de brise vers l'intérieur des terres, d'autre part, au sein de la région caennaise.

This paper proposed a part of a PhD work the aim of which is to know and understand the sea breezes to previous and prevent the ozone pollution in an urban coastal area.

A method of detection of sea breeze days has been developed to describe the main climatological features of sea breeze along the Calvados coast. The meteorological data of 1993-2002 of the station of Caen-Carpiquet (Météo-France) and the field measurement data of the summer 2003 define the frequency of occurrence of sea breezes days and the evolution of the front of the sea breeze in Caen area.

\section{INDEX}

Index géographique : Caen, Calvados, France

Mots-clés : brise de mer, littoral, pollution atmosphérique

Keywords : atmospheric pollution, littoral, sea breeze

\section{AUTEUR}

\section{AURÉLIE DUDOUIT}

GEOPHEN - Université de Caen Basse-Normandie, LETG-UMR 6554 - CNRS,

dudouit@geo.unicaen.fr 